\title{
Two-Dimensional DOA Estimation Using Arbitrary Arrays for Massive MIMO Systems
}

\author{
Alban Doumtsop Lonkeng and Jie Zhuang \\ School of Communication and Information Engineering, University of Electronic Science and Technology of China (UESTC), \\ Chengdu 611731, China \\ Correspondence should be addressed to Alban Doumtsop Lonkeng; doumtsopalban@yahoo.fr
}

Received 6 June 2017; Revised 8 August 2017; Accepted 6 September 2017; Published 17 December 2017

Academic Editor: Luciano Tarricone

Copyright ( $) 2017$ Alban Doumtsop Lonkeng and Jie Zhuang. This is an open access article distributed under the Creative Commons Attribution License, which permits unrestricted use, distribution, and reproduction in any medium, provided the original work is properly cited.

\begin{abstract}
With the quick advancement of wireless communication networks, the need for massive multiple-input-multiple-output (MIMO) to offer adequate network capacity has turned out to be apparent. As a portion of array signal processing, direction-of-arrival (DOA) estimation is of indispensable significance to acquire directional data of sources and to empower the 3D beamforming. In this paper, the performance of DOA estimation for massive MIMO systems is analyzed and compared using a low-complexity algorithm. To be exact, the 2D Fourier domain line search (FDLS) MUSIC algorithm is studied to mutually estimate elevation and azimuth angle, and arbitrary array geometry is utilized to represent massive MIMO systems. To avoid the computational burden in estimating the data covariance matrix and its eigenvalue decomposition (EVD) due to the large-scale sensors involved in massive MIMO systems, the reduced-dimension data matrix is applied on the signals received by the array. The performance is examined and contrasted with the 2D MUSIC algorithm for different types of antenna configuration. Finally, the array resolution is selected to investigate the performance of elevation and azimuth estimation. The effectiveness and advantage of the proposed technique have been proven by detailed simulations for different types of MIMO array configuration.
\end{abstract}

\section{Introduction}

The use of multiple antennas at the two finishes of wireless links is the consequence of the natural progression of more than four decades of advancement of adaptive antenna technology. Key administrations, for example, e-banking, e-learning, and e-well-being, will proceed to prosper and end up being more mobile. On-demand data and entertainment will steadily be conveyed over mobile and wireless communication systems. These expansions will prompt an avalanche of mobile and wireless traffic volume, anticipated to increase a thousandfold throughout the following decade [1]. Late advances have shown that multiple-inputmultiple-output (MIMO) wireless systems can accomplish great increments in the overall system performance. Such frameworks are expected for the development of new generations of mobile radio systems for future wireless communication standards and applications. Subsequently, it has gotten the consideration not just of the worldwide research and development community but also of the wireless communications industry [2]. There are several signal processing functions performed in MIMO systems, among which 3D beamforming for link reliability enhancement has gotten impressive consideration. To empower the 3D beamforming, precise estimation of both elevation and azimuth angles of signals is of key significance [3].

Over the last four decades, numerous high-resolution methods for estimating the DOA parameters of multiple narrow-band far-field signal sources, such as multiple signal classification (MUSIC), estimation of signal parameters via rotational invariance techniques (ESPRIT), weighted subspace fitting (WSF), and maximum likelihood (ML), have been proposed. A large number of these methods are applicable only to specific array geometries such as uniform linear 
arrays (ULAs), uniform circular arrays (UCAs), or uniform rectangular arrays (URAs). However, in practical cases, the limitations of an array platform usually lead to an unrealistic choice of array geometry from some specific classes. Furthermore, nonuniform array geometries are empowered to accomplish considerably enhanced resolution performance as compared to uniform array geometries with a similar number of sensors [4].

The automatic weighted subspace fitting (AWSF) algorithm for DOA estimation is demonstrated in [5]. The estimation accuracy is enhanced, but no implementation has already been done in MIMO systems to assess the performance. Algorithms based on sparse representative (SR) procedures $[6,7]$ deal with the computational complexity reduction of the $3 \mathrm{D} \mathrm{DOA}$ estimation and handle the issues of the assistant calibration for smart transportation frameworks, yet it is substantial for a predefined (URA, ULA, planar array, etc.) array configuration as a vast majority of DOA algorithms [8,9]. The DOA estimation task has been recast to a probabilistic system in $[8,9]$, identifying the smallest angular regions where the approaching signal is in all likelihood. Although effective for some applications, the method is insufficient for high-resolution analysis since spatially close signals cannot be properly sensed. Therefore, a multiresolution technique has been applied to improve the system [10]. Notwithstanding the positive and appealing highlights of previously mentioned approaches, every one of them shares a similar bottleneck. Undoubtedly, they need the assessment of the covariance matrix estimated from the measurements of every sensor at different snapshots. This includes a conspicuous increment in the receiver complexity and a delay in the DOA recovery [11]. In [12], a two-stage full-dimension DOA estimation scheme based on the MUSIC algorithm is given. It requires an exhaustive multidimensional peak search, resulting in a relatively high computational complexity as most 2D DOA estimation algorithms. The performance of DOA estimation for massive MIMO systems is assessed in [13] using a lowcomplexity algorithm; good performance was achieved, but this method is unsubstantial for arbitrary array structure. Therefore, development of low-complexity DOA estimation algorithms for massive MIMO systems in arbitrary array configuration becomes extremely necessary for a practical implementation perspective.

In this paper, 2D Fourier domain line search MUSIC (FDLSM) is introduced to jointly estimate elevation and azimuth information for massive MIMO systems. The motivation for applying this algorithm is threefold $[14,15]$.

(1) It reduces the computational complexity contrasted with the conventional 2D space search MUSIC or polynomial rooting strategies, by using $2 \mathrm{D}$ fast Fourier transform (2D FFT) that avoids the polynomial rooting step by replacing it with a computationally simple line search procedure.

(2) It provides an improved DOA estimation performance as compared to the manifold separation technique (MST).

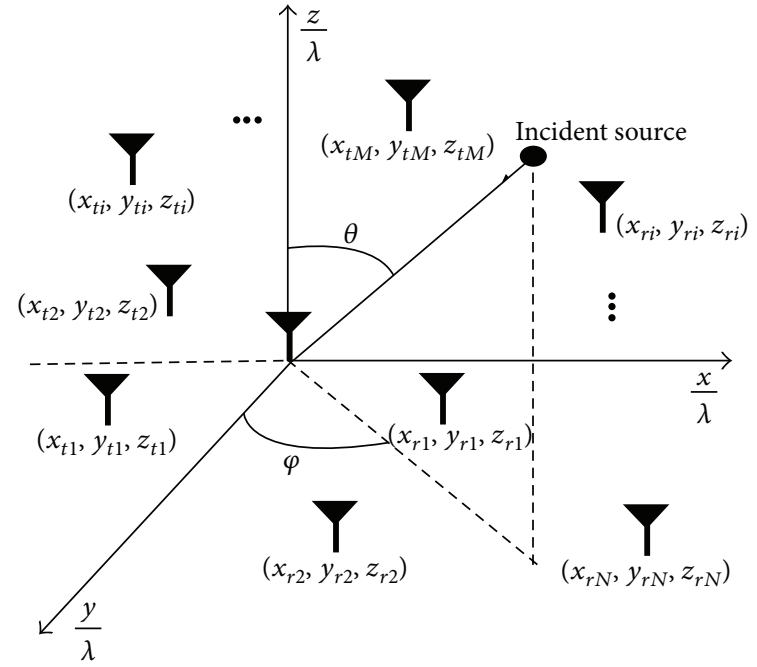

FIgURe 1: System model of 2D massive MIMO with an $\mathrm{MxN}$ arbitrary array configuration.

(3) It is formulated in terms of a reduced-dimension technique throughout the whole estimation process of the data covariance matrix, thus decreasing the computational burden of estimating the covariance matrix and its eigenvalue decomposition (EVD).

This paper fundamentally concentrates on a lowcomplexity 2D DOA estimation using the 2D FDLSM algorithm with a reduced-dimension transformation in massive MIMO systems. Signal-to-noise ratio (SNR), source number, and antenna configuration are chosen to assist the evaluation of algorithm performance. Moreover, to the best of our knowledge, 2D FDLSM is used for the first time to examine the elevation and azimuth estimation in massive MIMO systems.

The rest of this paper is sorted out as follows. The system model and 2D FDLSM are described in Section 2 and Section 3, respectively. The computer simulation and performance evaluation are presented in Section 4. Section 5 concludes the paper.

\section{System Model}

We consider a MIMO system equipped with $M$ transmit array sensors and $N$ receive array sensors at the base station. Both transmit and receive arrays are assumed to be closely located in the arbitrary space so that any target located in the far field can be seen at the same direction by both arrays. All the array sensors are omnidirectional. We assume that the number of incident sources is known and there exist $P$ distinct uncorrelated signals coming from directions of $\left(\theta_{p}, \phi_{p}\right), p=1,2, \ldots, P$, where $\theta_{p}$ and $\phi_{p}$ are the elevation and azimuth angle of the $p$ th signal, respectively. The system model is depicted in Figure 1. 
At the transmit side, $M$ transmit sensors from an $M$ sensor (arbitrary) array and its steering vector $\mathbf{a}_{\mathbf{t}}(\theta, \phi)$ is given by

$$
\mathbf{a}_{\mathbf{t}}\left(\theta_{p}, \phi_{p}\right)=\left[e^{j \frac{2 \pi}{\lambda}\left(x_{t 1} \sin \theta_{p} \cos \phi_{p}+y_{t 1} \sin \theta_{p} \sin \phi_{p}+z_{t 1} \cos \theta_{p}\right)}, \ldots, e^{\frac{j \pi}{\lambda}\left(x_{t M} \sin \theta_{p} \cos \phi_{p}+y_{t M} \sin \theta_{p} \sin \phi_{p}+z_{t M} \cos \theta_{p}\right)}\right]^{T}
$$

where $\lambda$ is the signal wavelength, $j=\sqrt{-1}$ and $\left(x_{t i}, y_{t i}, z_{t i}\right)$ are coordinates of the $i$ th transmit sensor, and $(\cdot)^{T}$ denotes the transpose.
For simplicity of notation and without loss of generality, we assume that the steering vector of the receive array is also a function of $(\theta, \phi)$, given by

$$
\mathbf{a}_{\mathbf{r}}\left(\theta_{p}, \phi_{p}\right)=\left[e^{j \frac{2 \pi}{\lambda}\left(x_{r 1} \sin \theta_{p} \cos \phi_{p}+y_{r 1} \sin \theta_{p} \sin \phi_{p}+z_{r 1} \cos \theta_{p}\right)}, \ldots, e^{j \frac{j \pi}{\lambda}\left(x_{r N} \sin \theta_{p} \cos \phi_{p}+y_{r N} \sin \theta_{p} \sin \phi_{p}+z_{r N} \cos \theta_{p}\right)}\right]^{T}
$$

where $\left(x_{r i}, y_{r i}, z_{r i}\right)$ are coordinates of the $i$ th receive sensor.

The output of the matched filters at the receiver is given by [16]

$$
\mathbf{x}(\tau)=\mathbf{A}(\theta, \phi) s(\tau)+\mathbf{n}(\tau)
$$

where $\mathbf{s}(\tau)=\left[s_{1}(\tau), s_{2}(\tau), \ldots, s_{p}(\tau)\right]^{T} \in C^{P \times 1}$ is the vector of signal waveforms with $\tau$ denoting the time interval, $\mathbf{n}(\tau)$ is the $B \times 1(B=M N)$ complex Gaussian white noise vector of the zero mean and covariance matrix $\sigma^{2} \mathbf{I}_{B}$, and $\mathbf{A}(\theta, \phi)=$ $\left[\mathbf{a}_{1}, \mathbf{a}_{2}, \ldots, \mathbf{a}_{\mathbf{p}}\right]$ is the $B \times P$ steering matrix composed of $P$ steering vectors with

$$
\begin{aligned}
\mathbf{a}_{\mathbf{p}} & =\mathbf{a}\left(\theta_{p}, \phi_{p}\right) \\
& =\mathbf{a}_{\mathbf{r}}\left(\theta_{p}, \phi_{p}\right) \otimes \mathbf{a}_{\mathbf{t}}\left(\theta_{p}, \phi_{p}\right),
\end{aligned}
$$

being the $B \times 1$ steering vector of the $p$ th signal. $\otimes$ represents the Kronecker product.

Assume that all impinging signals and noises are uncorrelated with each other. Then, the $B \times B$ data covariance matrix can be expressed as [16]

$$
\mathbf{R}_{\mathbf{x x}}=\frac{1}{L} \sum_{\ell=1}^{L} \mathbf{x}(\tau \ell) \mathbf{x}^{\mathbf{H}}(\tau \ell)
$$

where $L$ is the snapshot number and $(\cdot)^{H}$ stands for the Hermitian.

\section{2D DOA Estimation Using Arbitrary Arrays for Massive MIMO Systems}

In this section, a low-DOA estimation algorithm based on the FDLS MUSIC algorithm to jointly estimate the elevation and azimuth is presented.

3.1. Reduced-Dimension Beamspace (RDBS) Cramer-Rao Bound $(C R B)$. The length of the steering vector $\mathbf{a}_{\mathbf{p}}$ is $B$, which is too long and will add high computational burden. Furthermore, it will make the latter estimation of the covariance matrix in (5) and its EVD costly to implement because of the matrix size. So, the reduced-dimension transformation is necessary. It has been shown in [15] that there exist a transformation matrix $\mathbf{T}$ that makes $\mathrm{CRB}_{\mathrm{RDBS}}(\mathbf{T})=\mathrm{CRB}_{\mathrm{ESP}}$ (see (5) for ESP data), which is based on the following proven theorems [15]:

\section{Theorem 1}

$$
E\left[\{(\widehat{\theta}, \widehat{\phi})-(\theta, \phi)\}\{(\widehat{\theta}, \widehat{\phi})-(\theta, \phi)\}^{T}\right] \geq \mathrm{CRB}_{\mathrm{ESP}}
$$

where

$$
\mathrm{CRB}_{\mathrm{ESP}}=\frac{\sigma^{2}}{2 L}\left[\operatorname{Re}\left\{\left(\mathbf{W}^{\mathbf{H}} \mathbf{P}_{\mathbf{A}}^{\perp} \mathbf{W}\right) \odot\left(\mathbf{S A}^{\mathbf{H}} \mathbf{R}_{\mathbf{x x}}^{-1} \mathbf{A S}\right)^{T}\right\}\right]^{-1} .
$$

Here, $(\widehat{\theta}, \widehat{\phi}),(\theta, \phi), \mathbf{S}$, and $\odot$ denote the estimated DOAs, the true DOAs, the signal covariance matrix, and the Schur product, respectively.

$\mathbf{P}_{\mathbf{A}}^{\perp}=\mathbf{I}_{B}-\mathbf{A}\left(\mathbf{A}^{H} \mathbf{A}\right)^{-1} \mathbf{A}^{H}$ is the orthogonal projection onto the null space of $\mathbf{A}^{H}$ and

$$
\begin{aligned}
& \mathbf{W}=\left[\mathbf{w}\left(\theta_{1}, \phi_{1}\right), \ldots, \mathbf{w}\left(\theta_{P}, \phi_{P}\right)\right], \\
& \mathbf{w}\left(\theta_{p}, \phi_{p}\right)=\frac{\partial \mathbf{a}\left(\theta_{p}, \phi_{p}\right)}{\partial\left(\theta_{p}, \phi_{p}\right)}, \\
& p=1, \ldots, P .
\end{aligned}
$$

The expression of $\mathrm{CRB}_{\mathrm{RDBS}}(\mathrm{T})$ is obtained by substitution of $\mathbf{A}, \mathbf{W}$ and $\mathbf{R}_{\mathbf{x x}}$ with $\mathbf{T}^{\mathrm{H}} \mathbf{A}, \mathbf{T}^{\mathrm{H}} \mathbf{W}$, and $\mathbf{T}^{\mathrm{H}} \mathbf{R}_{\mathbf{x x}} \mathbf{T}$, respectively.

Theorem 2. Assume that $k \geq 2 P$ and that $\mathbf{T}$ is the $B \times k$ matrix fulfilling $\mathbf{T}^{H} \mathbf{T}=\mathbf{I}_{k}$. Then, if

$$
\Psi\{[\mathbf{A}(\theta, \phi) \mathbf{W}(\theta, \phi)]\} \subseteq \Psi\{\mathbf{T}\},
$$

the relation $\mathrm{CRB}_{\mathrm{RDBS}}(\mathrm{T},(\theta, \phi))=\mathrm{CRB}_{\mathrm{ESP}}((\theta, \phi))$ holds true. 
Here, $\Psi\{\cdot\}$ denotes the range space of the matrix and $\subseteq$ is read as "is contained in."

The proofs of the above theorems can be found in [15] and the references therein. After various simulations, $k=4 P$ was found to be the minimum value meeting the requirement in terms of accuracy.

According to [15], the matrix $\mathbf{T} \in \mathbb{C}^{B \times k}$ can be obtained by applying the singular value decomposition (SVD) of $\left[\begin{array}{ll}\mathbf{A} & \mathbf{W}\end{array}\right]$. Let

$$
\left[\begin{array}{ll}
\mathbf{A} & \mathbf{W}
\end{array}\right]=\mathbf{U} \boldsymbol{\Sigma} \mathbf{V}^{H}
$$

be the SVD of $\left[\begin{array}{ll}\mathbf{A} & \mathbf{W}\end{array}\right]$ for the $k$ largest singular values, where $\mathbf{U}$ is the $B \times k, \Sigma=\operatorname{diag}\left\{\varsigma_{1}, \ldots, \varsigma_{k}\right\}$, and $\left(\varsigma_{k} \geq \varsigma_{k} \geq \cdots \geq \varsigma_{k}\right)$ and $\mathbf{V}$ is the $k \times k$. Since $U$ spans the range space of $\left[\begin{array}{ll}\mathbf{A} & \mathbf{W}\end{array}\right]$, one may take $\mathbf{T}=\mathbf{U}$.

Include the $B \times k$ matrix $\mathbf{T}, P<k \leq B$, and the mapping $\mathbf{x} \mapsto \mathbf{y}=\mathbf{T}^{H} \mathbf{x}$ from ESP to RDBS, a new set of observation result, consisting of $k$-dimensional vectors. Then, in $R D B S$, the representation of the signals received by the array will be

$$
\begin{aligned}
\mathbf{y}(\tau) & =\mathbf{T}^{\mathbf{H}} \mathbf{x}(\tau) \\
& =\mathbf{T}^{\mathbf{H}} \mathbf{A}(\theta, \phi) \mathbf{s}(\tau)+\mathbf{T}^{\mathrm{H}} \mathbf{n}(\tau)
\end{aligned}
$$

For the signal model in (11), the covariance matrix $\mathbf{R}_{\mathbf{y}}$ can be estimated with $L$ snapshots by

$$
\mathbf{R}_{\mathbf{y y}}=\frac{1}{L} \sum_{\ell=1}^{L} \mathbf{y}\left(\tau_{\ell}\right) \mathbf{y}^{\mathbf{H}}\left(\tau_{\ell}\right)
$$

The eigen decomposition of the sample covariance matrix (12) yields

$$
R_{y y}=E_{s} \Lambda_{s} E_{s}^{H}+E_{n} \Lambda_{n} E_{n}^{H},
$$

where the sample eigenvalues are again sorted in descending order $\left(\lambda_{1} \geq \lambda_{2} \geq \cdots \geq \lambda_{k}\right)$; the matrices $\mathbf{E}_{\mathbf{s}} \cong\left[\mathbf{e}_{1}, \mathbf{e}_{2}, \ldots, \mathbf{e}_{\mathbf{p}}\right]$ and $\mathbf{E}_{\mathbf{n}} \cong\left[\mathbf{e}_{\mathbf{P}+\mathbf{1}}, \lambda_{\mathbf{P}+\mathbf{2}}, \ldots, \mathbf{e}_{\mathbf{k}}\right]$ contain in their columns the signal and noise subspace eigenvectors of (13), respectively. Correspondingly, the diagonal matrices $\Lambda_{\mathrm{s}} \cong \operatorname{diag}\left(\lambda_{1}, \lambda_{2}, \ldots\right.$, $\left.\lambda_{P}\right)$ and $\Lambda_{\mathbf{n}} \cong \operatorname{diag}\left(\lambda_{P+1}, \lambda_{P+2}, \ldots, \lambda_{k}\right)$ are built from the signal and noise subspace eigenvalues of (13), respectively.

3.2. 2D FDLS MUSIC-Based DOA Estimation. This section extends the idea of [14] into the 2D DFT to solve efficiently (14). The procedure is as follows:

By combining (4) and (13) and using the transformation matrix $\mathbf{T}$, the MUSIC null spectrum in [17] can be rewritten as

$$
\begin{aligned}
f(\theta, \phi) & =\left(\mathbf{T}^{\mathbf{H}} \mathbf{a}(\theta, \phi)\right){ }^{\mathbf{H}} \mathbf{E}_{\mathbf{n}} \mathbf{E}_{\mathbf{n}}^{\mathbf{H}}\left(\mathbf{T}^{\mathbf{H}} \mathbf{a}(\theta, \phi)\right) \\
& =\left(\mathbf{a}^{\mathbf{H}}(\theta, \phi) \mathbf{T}\right) \mathbf{E}_{\mathbf{n}} \mathbf{E}_{\mathbf{n}}^{\mathbf{H}}\left(\mathbf{T}^{\mathbf{H}} \mathbf{a}(\theta, \phi)\right) \\
& =\left\|\mathbf{E}_{\mathbf{n}}^{\mathbf{H}} \mathbf{T}^{\mathbf{H}} \mathbf{a}(\theta, \phi)\right\|^{2},
\end{aligned}
$$

where $\|\cdot\|$ is the vector 2-norm.

From (14), it can be seen that if $(\theta, \phi)$ is the desired DOA, $f(\theta, \phi)$ will be equivalent to zero. Given that $f(\theta, \phi)$ is a periodic function in $\theta$ and $\phi$ with the period $2 \pi$, it can be expressed using the finite 2D Fourier series as [18]

$$
f(\theta, \phi) \approx \sum_{m=-\left(Q_{e}-1\right)}^{Q_{e}-1} \sum_{n=-\left(Q_{a}-1\right)}^{Q_{a}-1} \mathbf{G}_{m n} e^{j m \theta} e^{j n \phi},
$$

where the Fourier coefficients are given by

$$
\mathbf{G}_{m n} \approx \frac{1}{4 \pi^{2}} \sum_{q_{e}=-\left(Q_{e}-1\right)}^{Q_{e}-1} \sum_{q_{a}=-\left(Q_{a}-1\right)}^{Q_{a}-1} f\left(k_{1}, k_{2}\right) e^{\left(-j m k_{1}\right)} e^{\left(-j n k_{2}\right)},
$$

with $k_{1}=q_{e} \Delta \theta, k_{2}=q_{a} \Delta \phi, \Delta \theta=2 \pi /\left(2 Q_{e}-1\right)$, and $\Delta \phi=2 \pi /$ $\left(2 Q_{a}-1\right)$, where $\left(2 Q_{e}-1\right)$ and $\left(2 Q_{a}-1\right)$ represent the sample number in elevation and azimuth, respectively.

We notice that 2D FFT can be used to obtain the Fourier coefficient matrix $\mathbf{G} \in C^{\left(2 Q_{e}-1\right) \times\left(2 Q_{a}-1\right)}$ rapidly. Once the Fourier coefficients are acquired, to ameliorate the resolution, zero padding is applied efficiently as follows:

$$
\tilde{\mathbf{G}}_{m n}=\left\{\begin{array}{l}
\mathbf{G}_{m n}, \quad \text { if }|m|<2 Q_{e}-1 \text { and } n \mid<2 Q_{a}-1, \\
0, \quad \text { if } 2 Q_{e}-1<|m|<J_{0} \text { and } 2 Q_{a}-1<|n|<J_{1} .
\end{array}\right.
$$

Generally, we pick $J_{0} \gg 2 Q_{e}-1$ and $J_{1} \gg 2 Q_{a}-1$. The new cost function $\tilde{f}(\theta, \phi)$ of every grid can be computed effectively by applying 2D IFFT to the zero padded Fourier coefficient matrix $\tilde{\mathbf{G}} \in C^{J_{0} \times J_{1}}$ in (17). Accordingly, no polynomial rooting is required any longer as the polynomial rooting step is replaced by a simple line search with $\theta$ and $\phi$.

Recall that $1 / \tilde{f}(\theta, \phi)$ will assume a very large value when $(\theta, \phi)$ is equal to the DOA of one of the signals. Thus, the $P$ signals can be easily obtained by taking the $P$ greatest peaks of $1 / \tilde{f}(\theta, \phi)$.

We also recall that the computational complexities of the conventional 2D space search MUSIC algorithm and the 2D FFT-based technique are entirely unequal. For the conventional 2D space search MUSIC algorithm, to obtain suitable accuracy, the intrigued area has to be divided into exceptionally dense grids. For instance, if the intrigued area is $\theta \in\left[0^{\circ}, 45^{\circ}\right]$ and $\phi \in\left[-50^{\circ}, 50^{\circ}\right]$ with $0.1^{\circ} \times 0.1^{\circ}$ as the unit grid, we have to compute $451 \times 1001=451451$ times the matrix-vector product $\left\|\mathbf{E}_{\mathbf{n}}^{\mathbf{H}} \mathbf{T}^{\mathbf{H}} \mathbf{a}(\theta, \phi)\right\|^{2}$ in (14). However, in the $2 \mathrm{D}$ FFT technique, only $\left(2 Q_{e}-1\right) \times\left(2 Q_{a}-1\right)$ matrixvector products in (14) need to be computed $\left(Q_{e}=Q_{a}=77\right.$ was used for our test). For other grids, zero padding is applied straightforwardly; hence, the computational complexity is decreased significantly. Furthermore, this technique has a lower complexity than the $2 \mathrm{D}$ polynomial rooting strategy. For instance, to estimate the 2D DOAs in [19], additional time is required to solve the high-order bivariate nonlinear polynomials. Since rooting a bivariate nonlinear polynomial is costly and the 2D FFT/IFFT method for real-time implementation is promptly accessible, the $2 \mathrm{D}$ FFT-based approach comes out to be competitive. 
TABle 1: Computational complexity.

\begin{tabular}{lcc}
\hline Algorithm & Using reduced-dimension matrix & Without using reduced-dimension matrix \\
\hline SVD & $O\left(\min \left(B^{2} k, B k^{2}\right)\right)$ & - \\
Covariance matrix & $O\left(k^{2} L+P^{3}\right)$ & $O\left(B^{2} L+P^{3}\right)$ \\
EVD & $O\left(k^{3}\right)$ & $O\left(B^{3}\right)$ \\
2D FDLS MUSIC & $O\left(Q_{e} Q_{a} k L+J_{0} J_{1} \log 2\left(J_{0} J_{1}\right)\right)$ & $O\left(Q_{e} Q_{a}(B) L+J_{0} J_{1} \log 2\left(J_{0} J_{1}\right)\right)$ \\
Total & $O(72,815,745)$ & $O(235,920,561)$ \\
\hline
\end{tabular}

TABle 2: Elapsed time of the two methods (100-simulation time, AMD E-450 APU $2 * 1.65 \mathrm{GHz}$, Windows 7 pro 64 bit, $2 \mathrm{~GB}$ memory, Matlab 8.4).

\begin{tabular}{lc}
\hline Method & Total time (seconds) \\
\hline Using reduced-dimension matrix & 49.471815 \\
Without using reduced-dimension matrix & 104.942036 \\
\hline
\end{tabular}

3.3. Summary. The major steps of the proposed algorithm for 2D DOA estimation in arbitrary geometry for the MIMO system are as follows:

(1) Obtain the RDBS matrix T from the SVD of (10) (for $k=4 P$ ); then, apply the mapping $\mathbf{x} \mapsto \mathbf{y}=\mathbf{T}^{H} \mathbf{x}$ to acquire the new representation of the array observations (11).

(2) From (11), construct the covariance matrix $\mathbf{R}_{\mathbf{y y}}$ in (12) and perform the eigenvalue decomposition to obtain the noise subspace $\mathbf{E}_{\mathbf{n}}$.

(3) Use $\left(2 Q_{e}-1\right) \times\left(2 Q_{a}-1\right)$ samples to compute the cost function in (14).

(4) Compute the Fourier coefficient matrix $\mathbf{G} \in$ $C^{\left(2 Q_{e}-1\right) \times\left(2 Q_{a}-1\right)}$ by applying the 2D FFT.

(5) Employ the zero padding technique described in (17) to shape the extensive matrix $\tilde{\mathbf{G}} \in C^{J_{0} \times I_{1}}$.

(6) Apply the 2D IFFT to $\tilde{\mathbf{G}}$ to obtain the new cost function $\tilde{f}(\theta, \phi)$.

(7) Estimate the desired DOAs from the null spectrum $1 / \tilde{f}(\theta, \phi)$ by taking the $P$ largest peaks.

3.4. Computational Complexity. The order of computational complexity of the proposed FD line search MUSIC for MIMO systems with a reduced-dimension matrix is compared with that of the case where the reduced-dimension matrix is not used. The results are shown in Table 1.

The last row of Table 1 shows the total number of operations (addition and multiplication) needed for both cases (i.e., $B=256, P=5, L=100$, and $J_{0}=J_{1}=2000$ ). We can easily see that, despite the fact that our approach uses the SVD, the computational complexity is reduced by $53 \%$ as compared with the case where the mapping matrix is not used.
TABLE 3: Simulation parameters.

\begin{tabular}{lc}
\hline Parameters & Setting \\
\hline Antenna configuration & Arbitrary array \\
Antenna elements & 256 \\
Center frequency & $3.0 \mathrm{Ghz}$ \\
Snapshots & 100 \\
Number of Monte Carlo & 100 \\
Directivity of the base station antennas & Omnidirectional
\end{tabular}

Table 2 gives the elapsed time by the two methods, from which it can be easily seen that the enhancement of the speed of our method is considerable.

\section{Simulation Results}

To get a more quantitative understanding of how the $2 \mathrm{D}$ Fourier domain (FD) line search MUSIC algorithm performs in massive MIMO systems, Monte Carlo simulations are conducted in this section. In the simulation, the total number of antenna elements is set to 256, which is considered one possible dimension of massive MIMO [1]. The case where there are 5 sources is used with $\phi=\left[-70^{\circ},-40^{\circ}\right.$, $\left.-13^{\circ}, 60^{\circ}, 80^{\circ}\right]$ and $\theta=\left[40^{\circ}, 31^{\circ}, 60^{\circ}, 70^{\circ}, 50^{\circ}\right]$. The simulation parameters are listed in Table 3.

Figure 2 shows the 2D spectrum of the null-spectrum function of arbitrary geometry $(\mathrm{SNR}=10 \mathrm{~dB})$ for different MIMO configurations with 256 antennas $(16 \times 16,64 \times 4$, $4 \times 64,32 \times 8$, and $8 \times 32$ ).

4.1. Performance Evaluation. The root-mean-squared error (RMSE) has been used to measure the effectiveness of our proposed 2D DOA estimation algorithm, which is defined as [13]

$$
\mathrm{RMSE}=\sqrt{\frac{1}{P} \frac{1}{N_{c}} \sum_{p=1}^{P} \sum_{n=1}^{N_{c}}\left[\left(\widehat{\theta}_{p, n}-\theta_{p}\right)^{2}+\left(\hat{\phi}_{p, n}-\phi_{p}\right)^{2}\right]},
$$

where $\widehat{\theta}_{p, n}$ is the estimated elevation angle $\theta_{p}$ at the $n$th Monte Carlo trial, which is similar for $\widehat{\phi}_{p, n}$. $P$ is the estimated source number and $N_{c}$ represents the Monte Carlo simulation times. The results are shown in Figure 3.

From the RMSE results for massive MIMO DOA estimation that are illustrated in Figure 3, we can see that the RMSE values of our proposed 2D FDLS MUSIC are 
2D FDLS MUSIC for MIMO

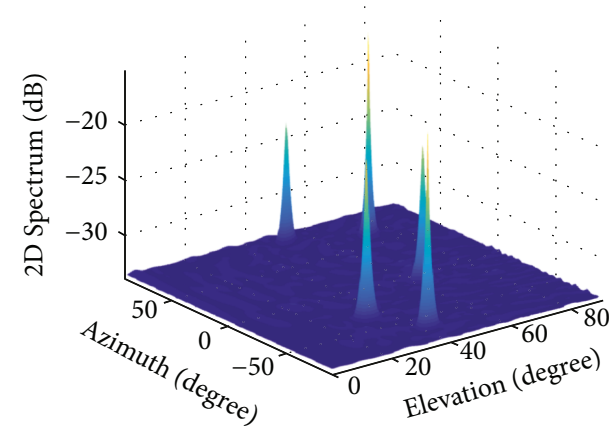

(a) $64 \times 4$ configuration

2D FDLS MUSIC for MIMO

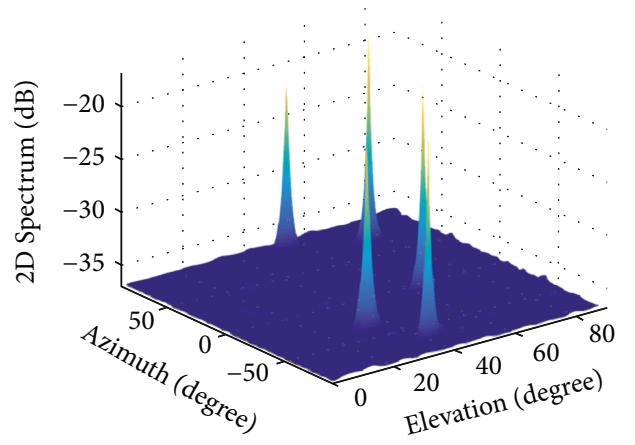

(c) $32 \times 8$ configuration
2D FDLS MUSIC for MIMO

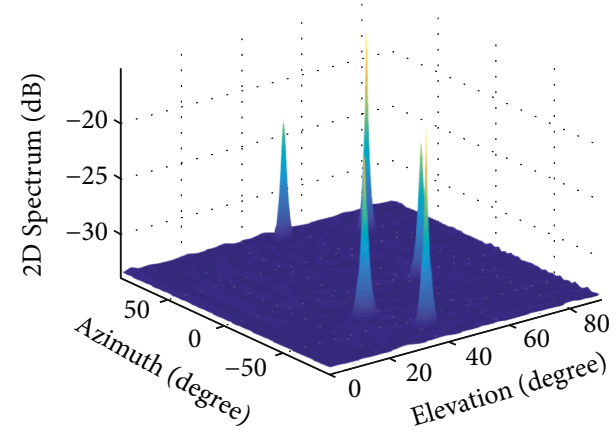

(b) $4 \times 64$ configuration

2D FDLS MUSIC for MIMO

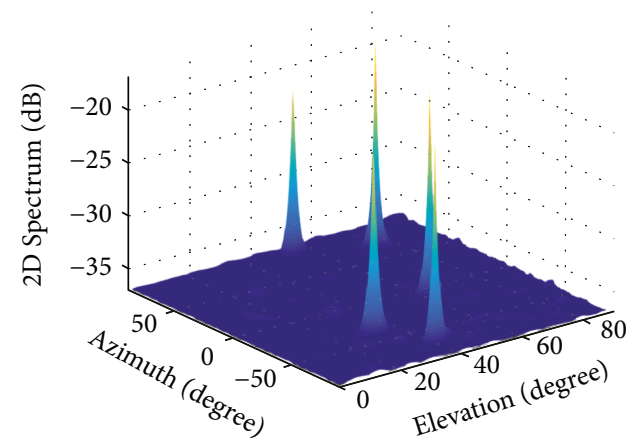

(d) $8 \times 32$ configuration

2D FDLS MUSIC for MIMO

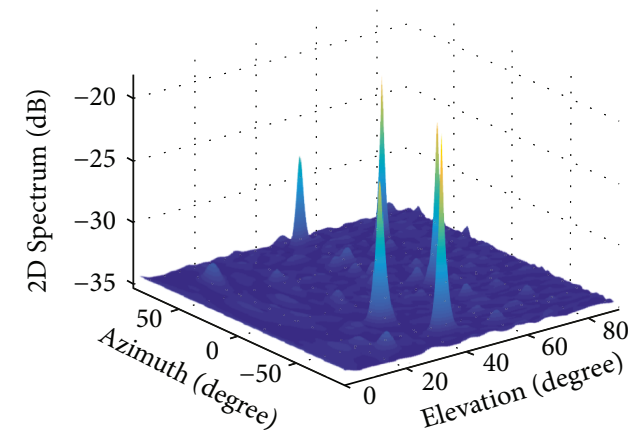

(e) $16 \times 16$ configuration

FIGURE 2: 2D FDLS MUSIC spectrum for MIMO in different antenna configurations.

below 0.065 with SNR ranging from $0 \mathrm{~dB}$ to $30 \mathrm{~dB}$. Furthermore, the DOA can be clearly observed; therefore, we can conclude that the proposed algorithm works well in massive MIMO systems. Different curves correspond to different types of antenna configuration as indicated in the legend. As the SNR increases, the performance of each array configuration becomes much more better. It is interesting to see that the $64 \times 4$ and $4 \times 64$ arrays have produced excellent quality compared with other array configurations.

Figure 2 also compares our proposed algorithm with the 2D MUSIC (using uniform circular array (UCA) structure). It shows that for SNR ranging from 10 to $30 \mathrm{~dB}$, both algorithms provide almost the same accuracy (for $64 \times 4,4 \times 64$, $8 \times 32$, and $32 \times 8$ configurations) while our approach uses less complexity as explained in Section 3.2.
From Figure 4, we can clearly observe that for the number of sensors varying from 50 to 256 elements, our approach still offers better performance than the traditional 2D MUSIC (UCA and $\mathrm{SNR}=20 \mathrm{~dB}$ were used).

4.2. Performance Comparison between Elevation and Azimuth Estimation. Figure 3 shows that two identical antenna configurations can have different performance. To better understand it, we have plotted the RMSE comparison between elevation and azimuth estimation of five sources with four types of array configuration (notice that $16 \times 16$ array configuration is not included due to its higher RMSE values); the results are shown in Figure 5.

It is easy to see from this figure that the overall performance of angle estimations weighs more in some cases while 


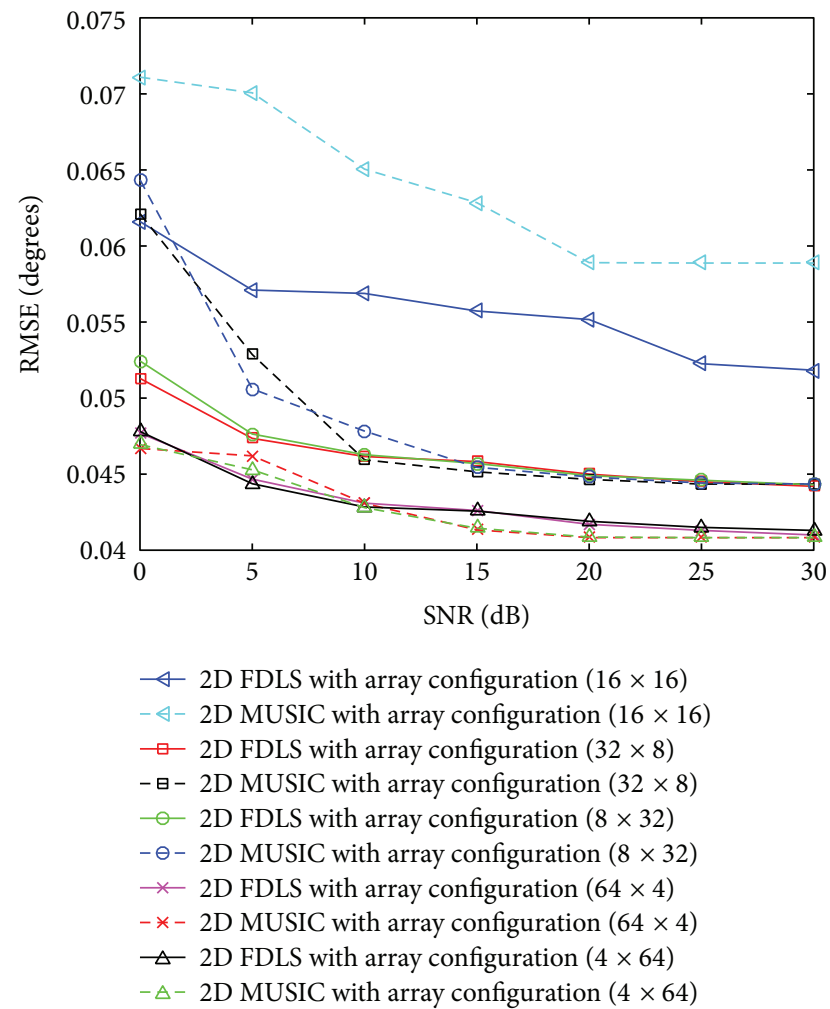

FIGURE 3: RMSE comparison between 2D MUSIC and 2D FDLS MUSIC algorithms for massive MIMO DOA estimation versus SNR for five sources with different types of array configuration.

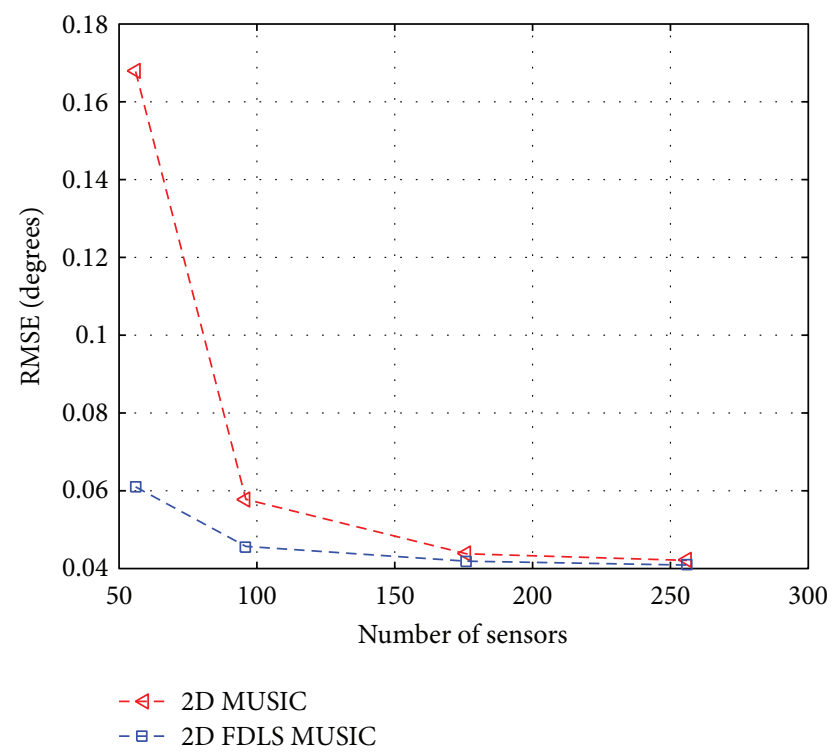

FIGURE 4: RMSE comparison between 2D MUSIC and 2D FDLS versus sensor number.

the accuracy of azimuth/elevation angles is more valued in other cases. Thus, the antenna array configuration can be adjusted as an efficient way to better satisfy the requirements of DOA estimation. Based on the simulations above, the

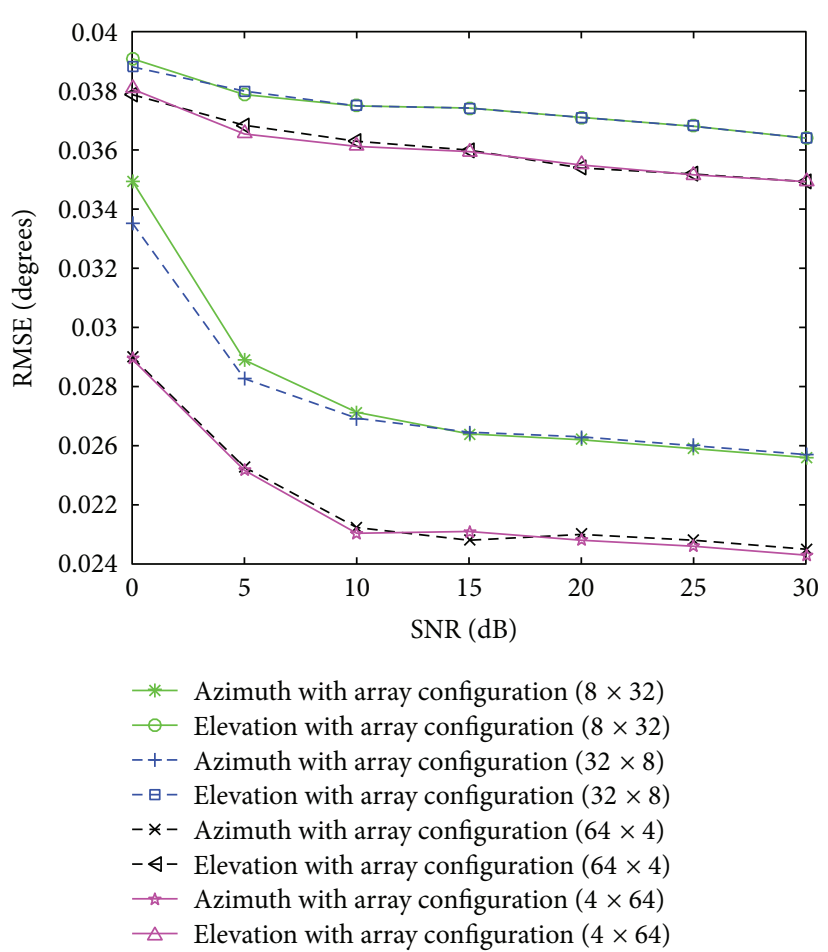

FIGURE 5: RMSE comparison between elevation and azimuth of five sources with eight types of array configuration.

$64 \times 4$ and $4 \times 64$ have the best performance of the overall DOA estimation. It is interesting to see that in the aspect of azimuth estimation, the $64 \times 4$ and $4 \times 64$ perform well while the $32 \times 8$ and $8 \times 32$ have good performance in elevation estimation. Figure 6 presents angle (azimuth and elevation) estimation results of our algorithm for all fives sources with $\mathrm{SNR}=10 \mathrm{~dB}$ using $64 \times 4$ configuration.

4.3. Performance Comparison between Array Configuration and Source Number. Figure 7 depicts the RMSE versus source number for $\mathrm{SNR}=10 \mathrm{~dB}$ with different types of sensor configuration. It shows that the RMSE increases gradually along with the growth of sources. Particularly, the $64 \times 4$ and $4 \times 64$ have the best performance.

4.4. Performance Comparison between Array Configuration and Snapshot Number. The RMSE for massive MIMO DOA estimation versus snapshot number for $\mathrm{SNR}=10 \mathrm{~dB}$ with different types of array configuration is plotted in Figure 8. It shows that the DOA estimation performance is getting better with the increase in the number of snapshots. That is because the error in estimating the signal covariance matrix in (12) is reduced with the increase in the snapshot number.

\section{Conclusion}

In this paper, a 2D DOA estimator in arbitrary array geometry for massive MIMO has been analyzed and modeled based on low-complexity 2D Fourier domain line 


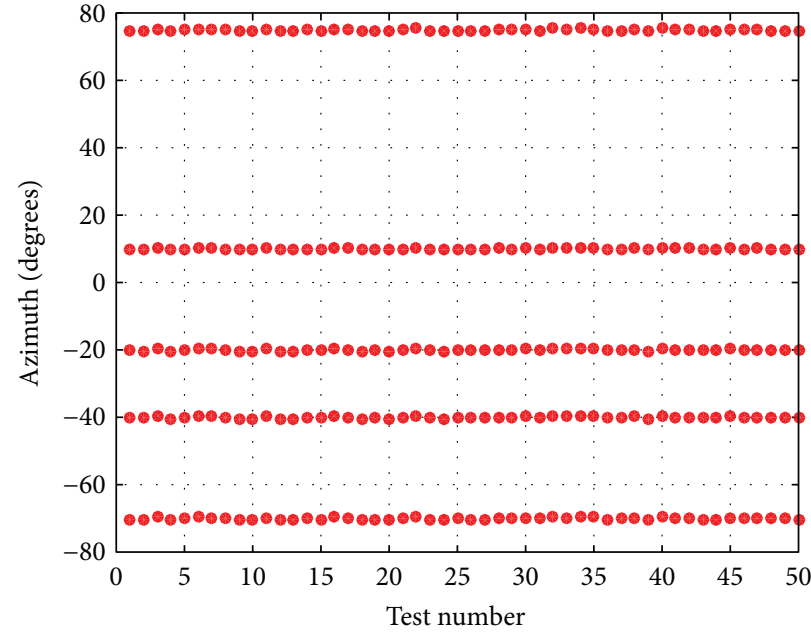

(a) Estimation results of azimuth angle

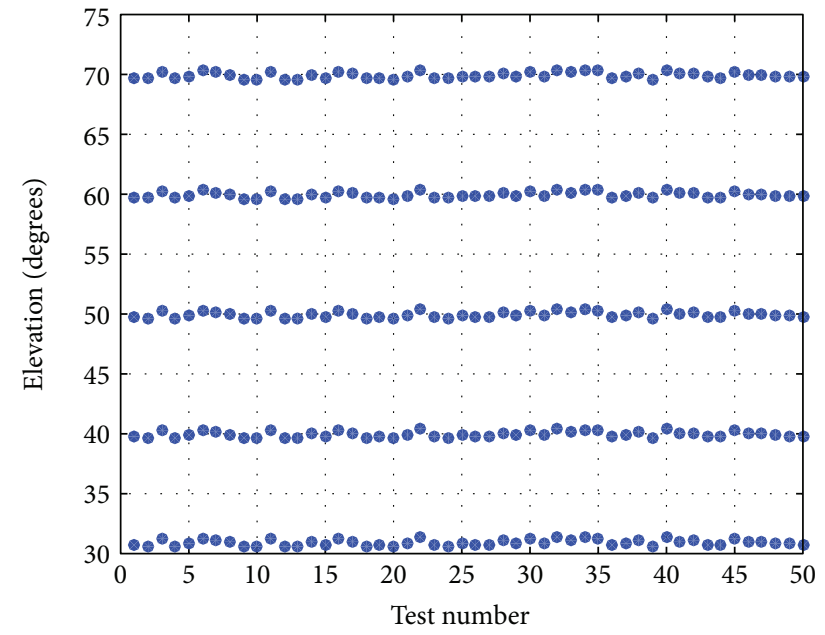

(b) Estimation results of elevation angle

FIgURE 6: Estimation results of DOA with $10 \mathrm{~dB}$ using $64 \times 4$ configuration.

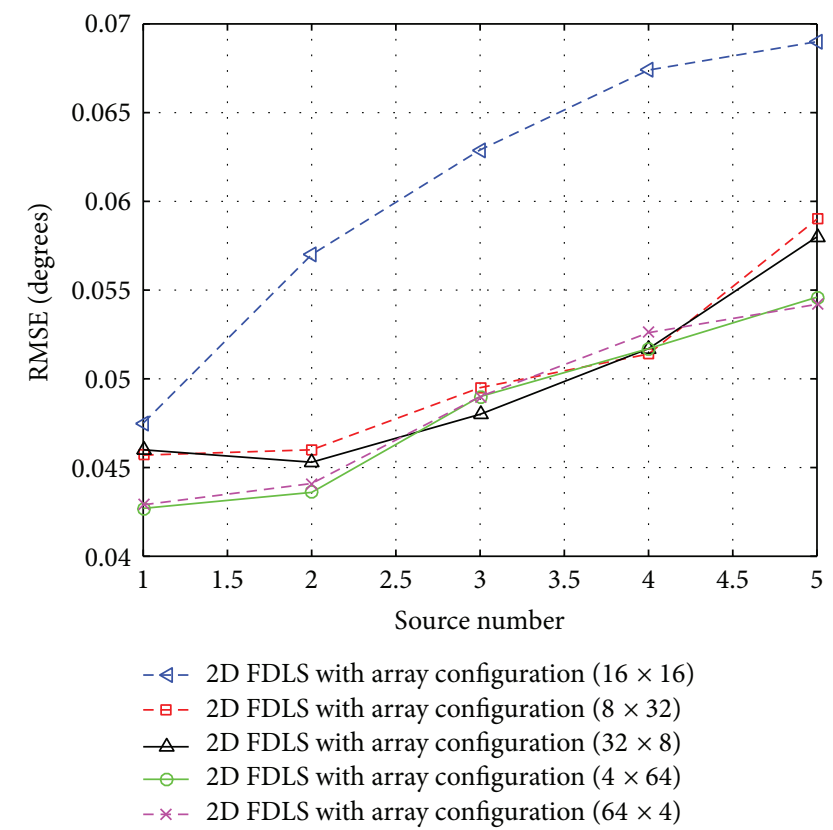

FIGURE 7: RMSE for massive MIMO DOA estimation versus source number for $\mathrm{SNR}=10 \mathrm{~dB}$ with different types of array configuration.

search MUSIC algorithm. The algorithm utilizes the 2D FFT to compute the null-spectrum function. It does not require a polynomial root-finding procedure to jointly estimate elevation and azimuth information, and it performs in a high quality in massive MIMO as shown in simulation results. Moreover, to avoid a large size of the covariance matrix and further decrease the computational complexity of its EVD, the reduced-dimension transformation has been applied on the received signals. The effectiveness and advantage of the proposed technique have been shown by detailed simulations for different types of MIMO array configuration.

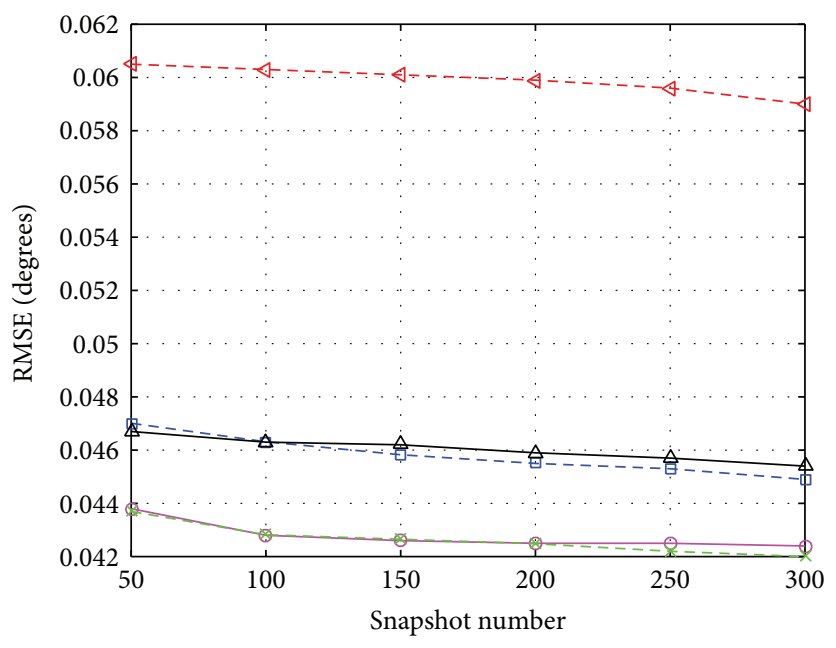

$-\triangleleft-2$ D FDLS with array configuration $(16 \times 16)$

$-\bullet-2$ D FDLS with array configuration $(8 \times 32)$

$\triangle 2$ 2D FDLS with array configuration $(32 \times 8)$

-2 D FDLS with array configuration $(4 \times 64)$

$-*-2$ D FDLS with array configuration $(64 \times 4)$

FIGURE 8: RMSE for massive MIMO DOA estimation versus snapshot number for $\mathrm{SNR}=10 \mathrm{~dB}$ with different types of array configuration.

\section{Conflicts of Interest}

The authors declare that they have no conflicts of interest.

\section{Acknowledgments}

This work was supported in part by the National Natural Science Foundation of China (NSFC) under Grant 61571090 and the Fundamental Research Funds for the Central Universities under Grant ZYGX2014J007. 


\section{References}

[1] A. Osseiran, F. Boccardi, V. Braun et al., "Scenarios for 5G mobile and wireless communications: the vision of the METIS project," IEEE Communications Magazine, vol. 52, no. 5, pp. 26-35, 2014.

[2] G. Tsoulos, MIMO Systems Technologies for Wireless Communications, Taylor \& Francis, Boca Raton, FL, USA, 1st edition, 2006.

[3] H. Halbauer, S. Saur, J. Koppenborg, and C. Hoek, "3D beamforming: performance improvement for cellular networks," Bell Labs Technical Journal, vol. 18, no. 2, pp. 37-56, 2013.

[4] A. B. Gershman, M. Rübsamen, and M. Pesavento, "One- and two-dimensional direction-of-arrival estimation: an overview of search-free techniques," Signal Processing, vol. 90, no. 5, pp. 1338-1349, 2010.

[5] H. Chen, Z. Pan, L. Tian, J. Shi, G. Yang, and M. Suzuki, "A novel AWSF algorithm for DOA estimation in virtual MIMO systems," IEEE Journal on Selected Areas in Communications, vol. 31, no. 10, pp. 1994-2003, 2013.

[6] G. Zhao, G. Shi, F. Shen, X. Luo, and Y. Niu, "A sparse representation-based DOA estimation algorithm with separable observation model," IEEE Antennas and Wireless Propagation Letters, vol. 14, pp. 1586-1589, 2015.

[7] P. Rocca, M. A. Hannan, M. Salucci, and A. Massa, "Singlesnapshot DOA estimation in array antennas with mutual coupling through a multiscaling BCS strategy," IEEE Transactions on Antennas and Propagation, vol. 65, no. 6, pp. 32033213, 2017.

[8] D. Malioutov, M. Çetin, and A. S. Willsky, "A sparse signal reconstruction perspective for source localization with sensor arrays," IEEE Transactions on Signal Processing, vol. 53, no. 8, pp. 3010-3022, 2005.

[9] G. Han, L. Wan, L. Shu, and N. Feng, "Two novel DOA estimation approaches for real-time assistant calibration systems in future vehicle industrial," IEEE Systems Journal, vol. 11, no. 3, pp. 1361-1372.

[10] M. Donelli, F. Viani, P. Rocca, and A. Massa, "An innovative multiresolution approach for DOA estimation based on a support vector classification," IEEE Transactions on Antennas and Propagation, vol. 57, no. 8, pp. 2279-2292, 2009.

[11] M. Carlin, P. Rocca, G. Oliveri, F. Viani, and A. Massa, "Directions-of-arrival estimation through Bayesian compressive sensing strategies," IEEE Transactions on Antennas Propagation, vol. 61, no. 7, pp. 3828-3838, 2013.

[12] K. Yang, J. Wu, and W. Li, "A low-complexity direction-ofarrival estimation algorithm for full-dimension massive MIMO systems," in 2014 IEEE International Conference on Communication Systems, pp. 472-476, Macau, China, 2014.

[13] T. Wang, B. O. Ai, R. He, and Z. Zhong, "Two-dimension direction-of-arrival estimation for massive MIMO systems," IEEE Access, vol. 3, pp. 2122-2128, 2015.

[14] M. Rübsamen, S. Member, and A. B. Gershman, "Direction-ofarrival estimation for nonuniform sensor arrays: from manifold separation to Fourier domain MUSIC methods," IEEE Transactions On Signal Processing, vol. 57, no. 2, pp. 588-599, 2009.

[15] S. Anderson, "On optimal dimension reduction for sensor array signal processing," Signal Processing, vol. 30, no. 2, pp. 245-256, 1993.
[16] W. Zhang, W. Liu, S. Wu, and J. Wang, "Robust DOA estimation for a MIMO array using two calibrated transmit sensors," in 2013 IET International Radar Conference, Xi'an, China, 2013.

[17] R. O. Schmidt, "Multiple emitter location and signal parameter estimation," IEEE Transactions on Antennas Propagation, vol. 34, no. 3, pp. 276-280, 1986.

[18] P. Yang, F. Yang, Z. P. Nie, H. J. Zhou, B. Li, and X. F. Tang, "Fast 2-D DOA and polarization estimation using arbitrary conformal antenna array," Progress in Electromagnetics Research C, vol. 25, no. 2011, pp. 119-132, 2012.

[19] M. Costa, V. Koivunen, and A. Richter, "Low complexity azimuth and elevation estimation for arbitrary array configurations," in 2009 IEEE International Conference on Acoustics, Speech and Signal Processing, pp. 2185-2188, Taipei, Taiwan, 2009. 


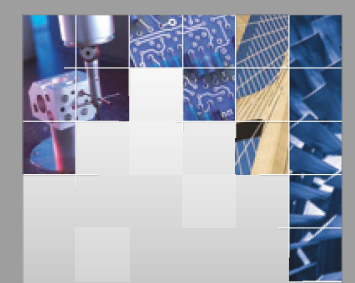

\section{Enfincering}
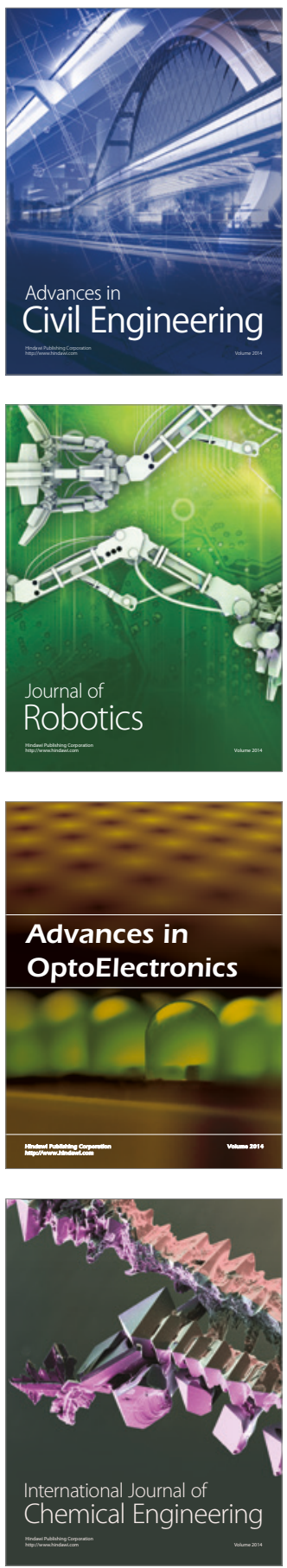

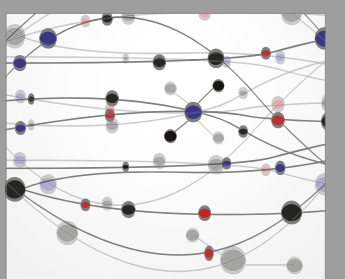

The Scientific World Journal

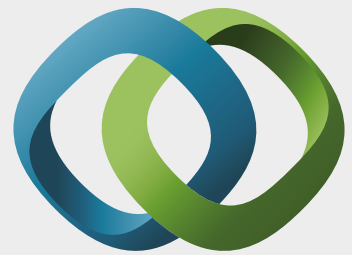

\section{Hindawi}

Submit your manuscripts at

https://www.hindawi.com
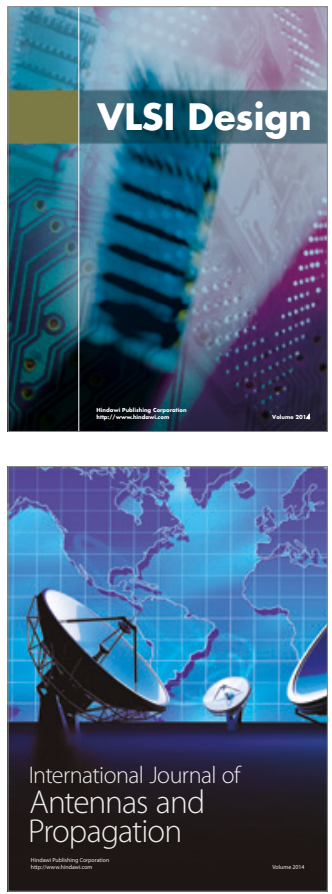

\section{Rotating}

Machinery
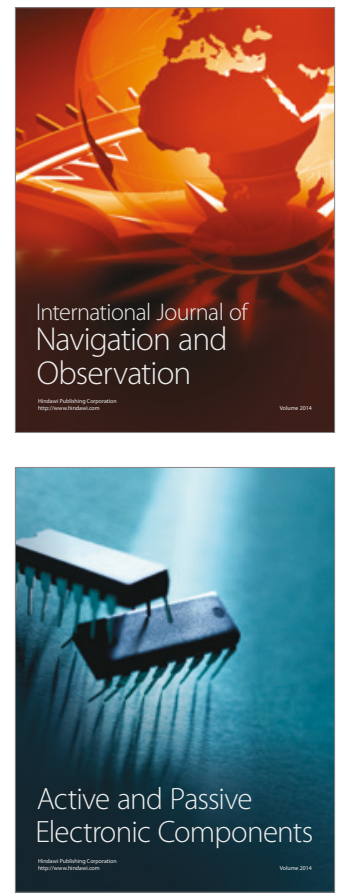
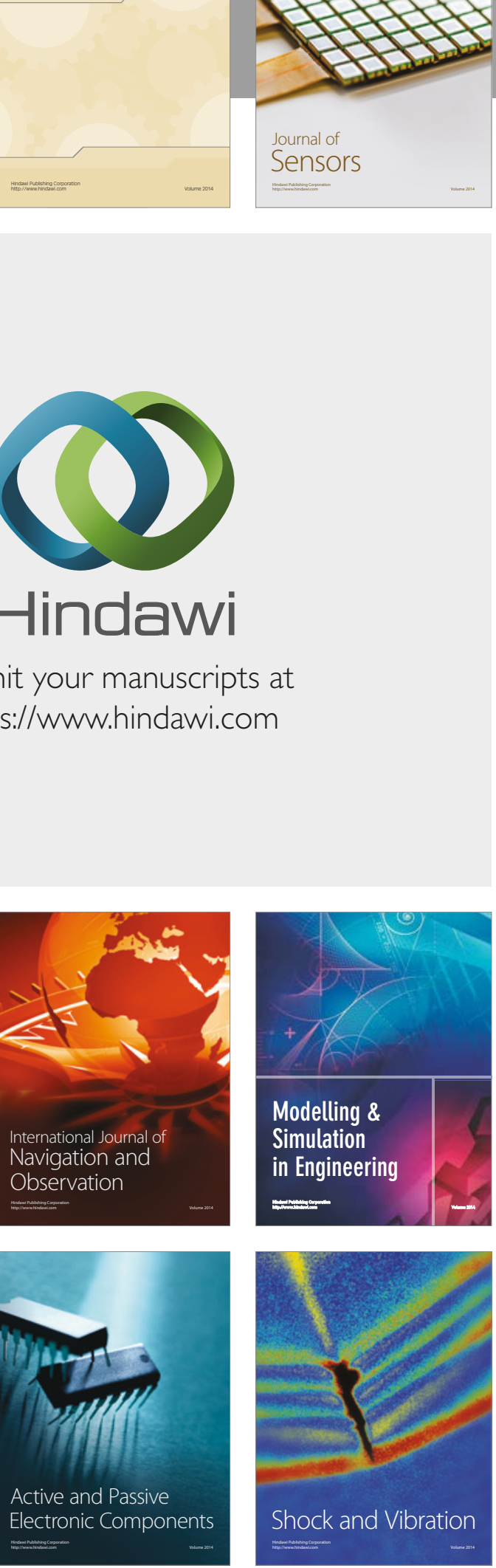
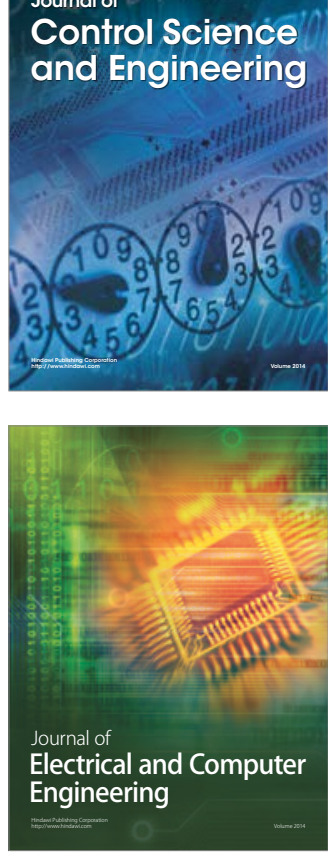

Distributed

Journal of

Control Science

and Engineering
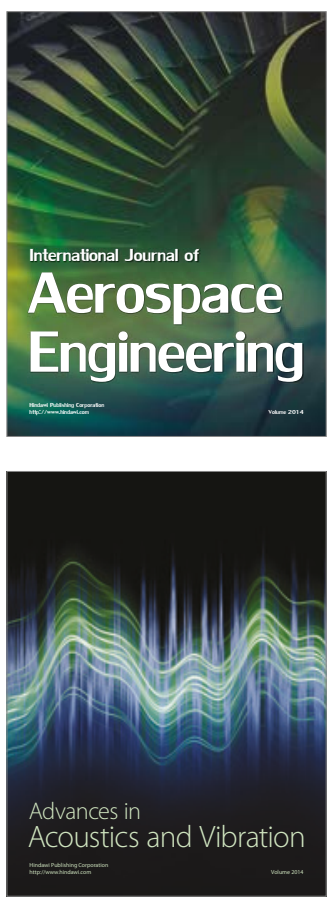

Sensor Networks 